\title{
Multiple Stress Fractures: A Case Report
}

\author{
Michail Kokkinakis ${ }^{\mathrm{a}, \mathrm{b}}$, Spyridon Darmanis ${ }^{\mathrm{a}}$, Stefanos Anastasopoulos ${ }^{\mathrm{a}}$, Ioannis Zagkas ${ }^{\mathrm{a}}$, \\ Konstaninos Karliaftis ${ }^{\mathrm{a}}$
}

\begin{abstract}
The stress fracture is a common injury sustained in the majority of the cases as a solitary injury in the tibia, fibula and metatarsals. Clinical examination of such injuries is often non-specific and early plain radiographs may be inconclusive. The prevention has proved to be difficult. We report a case of an eighteen-year-old female military trainee who presented with synchronous multiple stress fractures sustained in the lower limbs within one month from the start of her military training. A review of the literature with regards to epidemiology, signs, diagnosis, treatment, and prevention of such injuries is carried out and high risk individuals are outlined.
\end{abstract}

Keywords: Multiple stress fractures; Military; Prevention

\section{Introduction}

Stress fracture is an overuse injury caused by muscle forces together with bending and impact forces acting on the bone, which has not adapted to the loading. The reliable diagnosis of stress fractures is often difficult, as the radiographs are not usually positive until 2 weeks after the onset of symptoms and further imaging is required. Tibia, fibula, and metatarsals are the usual bones found, and early plain radiographs may be inconclusive. The prevention has proved to be difficult. We report a case of an eighteen-year-old female military trainee who presented with synchronous multiple stress fractures sustained in the lower limbs within one month from the start of her military training.

\footnotetext{
Manuscript accepted for publication September 29, 2010

${ }^{a}$ Department of Trauma \& Orthopaedics, 401 General Military Hospital, Athens, Greece

${ }^{\mathrm{b}}$ Corresponding author: 14 Makrigianni Street, Gerakas, Athens, 15344, Greece. Email: mkokkinakis@doctors.org.uk
}

doi:10.4021/jmc46w

\section{Case Report}

An 18-year-old female military trainee presented in the orthopaedic fracture clinic of 401 General military hospital in Athens with a 1-month history of generalized pain to bilateral lower limbs since she started the basic military training. She also reported 2 episodes of inversion injuries to both feet while jogging. She was otherwise fit and healthy and did not receive any medication. Clinical examination revealed pain on palpation on bilateral mid-thighs, forefeet, and right mid-shin. The patient was able to weight bear on both lower limbs with a normal gait. Plain radiographs demonstrated recent multiple stress fractures with still visible fracture lines and beginning callus formation in all painful limbs. Consequently, a bone scan confirmed the above radiological diagnosis and ruled out any other areas of osseous pathology (Fig. 1).

A DEXA scan revealed normal bone density. All biochemical and laboratory tests were unremarkable including urea and electrolytes, creatinine, full blood count, calcium and phosphor, and complete endocrinological testings (PTH, TSH, ACTH, prolactin, LH). Vitamin D levels were also normal.

\section{Discussion}

Stress fractures result from repetitive, cyclic loading of bone which overwhelms the reparative ability of the skeletal system. Mechanically, three events may lead to stress fractures. First, the applied load can be increased. Secondly, the number of applied stresses can increase. Finally, the surface area

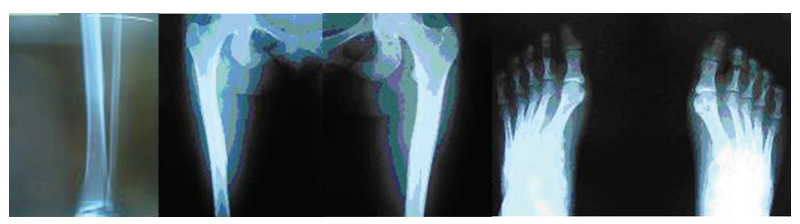

Figure 1. Multiple stress fractures demonstrated in tibia shaft, bilateral femoral shafts, 2nd and 3rd metatarsals. 
over which the load is applied can be decreased [1].

A number of predisposing factors have been identified, related to bone dimensions and the characteristics of the force applied. Bending is the most important force involved in their development, rather than torsion, tensile, and compressive forces. Bending forces are influenced by the properties and type of collagen, and the geometrical distribution of bone mass, rather than its absolute value. Hence, it is not surprising that measurements of bone mineral content (BMC) and density (BMD) may not identify individuals at risk of stress fractures [2].

The lower limb is the major site for stress fractures, particularly of the tibia, fibula, calcaneum, and metatarsals. The tibia is reported to be the most common site, representing up to 64 percent of stress fractures, while the metatarsals represent 21 percent. Femoral stress fractures are less common and can be divided into those involving the femoral neck, condyles, or shaft. Those of the femoral shaft are reported to comprise between 2.8 and 21 percent of femoral stress fractures in athletes [3]. Stress fracture is an overuse injury caused by muscle forces together with bending and impact forces acting on the bone, which has not adapted to the loading. The posteromedial area of the femoral shaft has been shown to have the greatest strain in the sagittal plane, but is also susceptible to stress fracture due to the origin of the vastus medialis and the insertion of the adductor brevis. In cases of overuse and elevated bone stress, it is suspected that either muscle fatigue leads to a decrease in the ability to absorb shock, and/or highly concentrated forces act through small areas of tendinous insertion to overload bone [4].

Specific risk factors for stress fractures may exist in certain individuals and for specific fracture sites. Anatomical features as predisposing factors have been identified including short and/or narrow long bones or external rotation of the hip and general factors, including gender, race, and age. Stress fractures occur more frequently in female athletes in relation to their male counterparts. There is a demonstrated relationship to eating disorders, amenorrhea, and osteoporosis, or the female athlete triad. Thus, stress fractures in the female athlete require additional investigation into those areas [1]. Sequential but not synchronous bilateral femoral fatigue fractures have been associated with the long term use of biphosphonates [5]. There is limited evidence for factors such as body habitus, training surfaces, and footwear to increase the risk for stress fractures. There is no association of appropriate vegan diets and occurrence of stress fractures in the literature.

In the history of these patients, a considerable amount of running exercise is usually found. The symptoms are stress pain and aching at rest after training. Typical findings are local palpation pain and edema. Sometimes tender resistance is felt. Overall the symptoms are vague, particularly in femoral stress fractures. Specific physical examination techniques can assist in diagnosis but require a very high index of sus- picion. Initial plain radiological evaluation may be normal, especially early in the course of a stress fracture. Delayed radiographs reveal the presence of a periosteal reaction which often mimics neoplastic lesions. Further radiological evaluation may be necessary to make a definitive diagnosis. Repeating plain radiographs, bone scintigraphy, magnetic resonance imaging, and computerized tomography are all possible options.

The corner stones of the treatment are: early identification of the symptoms, early diagnosis, a sufficiently long training pause, and in special cases consultation of experts in the field. Treatment consists of decreased activity to painfree levels and painkillers. Low-impact conditioning such as swimming and bicycling is allowed. Hamstring and quadriceps stretching is suggested. Treatment options begin with rest and cessation of the precipitating activity. Femoral stress fractures represent a small subset of lower extremity stress fractures but can have grave consequences if undetected, resulting in a displaced fracture. If detected prior to displacement, conservative treatment of these fractures is highly successful. However, displacement frequently makes surgical treatment necessary. In case of bilateral symptoms, resulting in inability to mobilize without weight bearing, or inability to refrain from weight bearing for any other reason, preventive nailing should be considered [6]. Biphosphonates should not be used to treat stress fractures. Our patient was treated conservative and with the corresponding rehabilitation she returned to her military duties three months later without further orthopaedic problems reported.

Niva et al performed a study and reported of bone stress injuries at femur and tibia which were asymptomatic at military recruits during intensive training and did not recommend routine screening of asymptomatic military trainees [7]. A systematic review by Snyder et al showed evidence of shoe insoles to reduce the overall femoral and tibial stress fracture incidence during military training by improving biomechanics, lessening fatigue, and attenuating impact. According to the same review, it remained unclear if the use of insoles would prevent stress fractures in athletes [8]. Ruohola et al showed elevated serum tartrate-resistant acid phosphatase isoform 5b (TRACP-5b) levels to reflect enhanced bone remodeling as in cases of stress fractures in military conscripts but failed statistically with his study to give a conclusive statement of its usefulness as a diagnostic tool [9]. High suspicion to further imaging should be considered in individuals with endocrine disorders malalignment, nutritional deficiencies, and endocrine disorders.

In conclusion, diagnosis requires thorough clinical evaluation with a high index of suspicion for stress fractures. History must focus on examining the military trainees' activities, especially any changes in running/walking distance, running surface, and type of shoe. The diagnosis and treatment of stress fractures is a challenge for the physician. It requires a high index of suspicion combined with a strong 
knowledge of the at-risk stress fractures and their complications. Accurate and timely diagnosis is required to prevent possible costly and disabling complications. Early referral for MRI or bone scan is recommended, to aid prompt diagnosis and treatment and to prevent more serious sequelae.

\section{References}

1. Reeder MT, Dick BH, Atkins JK, Pribis AB, Martinez JM. Stress fractures. Current concepts of diagnosis and treatment. Sports Med 1996;22(3):198-212.

2. Giladi M, Milgrom C, Simkin A, Danon Y. Stress fractures. Identifiable risk factors. Am J Sports Med 1991;19(6):647-652.

3. Matheson GO, Clement DB, McKenzie DC, Taunton JE, Lloyd-Smith DR, MacIntyre JG. Stress fractures in athletes. A study of 320 cases. Am J Sports Med 1987;15(1):46-58.

4. Weind KL, Amendola A. Rare bilateral femoral shaft stress fractures in a female long-distance runner: a case report. Iowa Orthop J 2005;25:157-159.

5. Capeci CM, Tejwani NC. Bilateral low-energy simultaneous or sequential femoral fractures in patients on long-term alendronate therapy. J Bone Joint Surg Am 2009;91(11):2556-2561.

6. Somford MP, Geurts GF, den Teuling JW, Thomassen BJ, Draijer WF. Long-Term Alendronate Use Not without Consequences? Int J Rheumatol 2009;2009;253432.

7. Niva MH, Mattila VM, Kiuru MJ, Pihlajamaki HK. Bone stress injuries are common in female military trainees: a preliminary study. Clin Orthop Relat Res 2009;467(11):2962-2969.

8. Snyder RA, DeAngelis JP, Koester MC, Spindler KP, Dunn WR. Does shoe insole modification prevent stress fractures? A systematic review. HSS J 2009;5(2):92-98.

9. Ruohola JP, Mulari M, Haataja RI, Vaananen HK, Pihlajamaki HK. Can elevated serum TRACP-5b levels predict stress fractures? A cohort study. Scand J Surg 2009;98(4):239-243. 